\title{
Clinical Frailty Scale em idosos atendidos no Serviço Hospitalar de Emergência: a fragilidade basal é um bom preditor de mortalidade em 90 dias?
}

\author{
Clinical Frailty Scale in older adults admitted at Emergency Department: is baseline frailty a good \\ predictor of Ninety-Day Mortality?
}

\author{
Tatiana de Carvalho Espíndola Pinheiro',2,3 $\mathbb{E}$ \\ Camila Oliveira Alcântara ${ }^{2,3}$ (DD \\ Fabiano Moraes Pereira ${ }^{2,3}$ (D) \\ Marcus Vinícius Melo de Andradel, 1,5 (ID \\ Edgar Nunes de Moraes 2,4 (DD \\ Maria Aparecida Camargos Bicalho $0^{1,2,4}$ (D)
}

\section{Resumo}

Objetivo: Avaliar a capacidade da Clinical Frailty Scale (CFS) em predizer a mortalidade em até 90 dias e outros desfechos desfavoráveis em idosos admitidos em um Serviço Hospitalar de Emergência (SHE). Método: Estudo de coorte prospectivo que incluiu idosos admitidos e que permaneceram por pelo menos uma noite no SHE de um hospital público terciário. O grau de fragilidade basal foi avaliado através da CFS e sua pontuação, o preditor estudado, por meio da curva Receiver Operator Characteristics (ROC). Analisou-se como desfecho primário a mortalidade em 90 dias. Considerou-se como desfechos secundários: mortalidade em 180 dias, declínio funcional, readmissão no SHE, reinternação e necessidade de atenção domiciliar. Resultados: 206 participantes foram incluídos. Dos 127 idosos frágeis, 40 (31,5\%) faleceram até o $90^{\circ}$ dia comparado a $5(6,3 \%)$ do grupo não frágil $(p<0,001)$. Após ajuste para variáveis demográficas e clínicas, a fragilidade manteve-se no modelo como um preditor independente de mortalidade em 90 dias da admissão. A acurácia obtida pela curva ROC (AUROC) para predição de mortalidade em 90 dias foi de 0,81. Para mortalidade em 180 dias foi 0,80; para necessidade de atenção domiciliar, 0,77; e para reinternação, 0,65. Para os demais desfechos estudados, a acurácia não foi significativa. Conclusão: A fragilidade basal medida pela CFS é um bom preditor de mortalidade em 90 e 180 dias e de necessidade de atenção domiciliar em idosos admitidos no SHE. Sua aplicação nesse cenário pode auxiliar na tomada de decisões clínicas.

\footnotetext{
Universidade Federal de Minas Gerais, Faculdade de Medicina, Programa de Pós-Graduação em Ciências Aplicadas à Saúde do Adulto. Belo Horizonte, MG, Brasil.

2 Universidade Federal de Minas Gerais, Hospital das Clínicas, Serviço de Geriatria e Gerontologia. Belo Horizonte, MG, Brasil.

3 Universidade Federal de Minas Gerais, Hospital das Clínicas, Equipe de Cuidados Paliativos. Belo Horizonte, MG, Brasil.

4 Universidade Federal de Minas Gerais, Faculdade de Medicina, Departamento de Clínica Médica. Belo Horizonte, MG, Brasil.

5 Universidade Federal de Minas Gerais, Hospital das Clínicas, Serviço de Apoio Diagnóstico e Tratamento em Medicina de Emergência. Belo Horizonte, MG, Brasil.
}

Os autores declaram não haver conflito na concepção deste trabalho.

Financiamento da pesquisa: Coordenação de Aperfeiç,oamento de Pessoal de Nível Superior - Brasil (CAPES)

- Código de Financiamento 001.
Palavras-chave: Fragilidade. Envelhecimento. Fatores de Risco. Mortalidade. Serviço Hospitalar de Emergência. 


\section{Abstract}

Objective: To evaluate the ability of the Clinical Frailty Scale (CFS) to predict 90-day mortality and other poor outcomes in older adults admitted at a Hospital Emergency Department (ED). Method: This is a prospective cohort study including older adults admitted at ED of a Public Hospital who spent at least one night in it. The degree of baseline frailty was assessed through the CFS, and its score was the predictor studied, through the Receiver Operator Characteristics (ROC) curve analysis. We analyzed 90-day mortality as a primary outcome. The following outcomes were considered as secondary ones: mortality, functional decline, readmittance to $\mathrm{ED}$, readmission and need for home care. Results: 206 participants were included. Of the 127 frail older adults, 40 (31.5\%) died before the 90th day compared to $5(6.3 \%)$ in the non-frail group $(\mathrm{p}<0.001)$. After adjustment for demographic and clinical variables, frailty remained in the model as an independent predictor of 90-day mortality. The accuracy obtained by the ROC curve (AUROC) for predicting 90 -day mortality was 0.81 . For 180 -day mortality, 0.80 , for the need for home care, 0.77 for readmission, 0.65 . For the other outcomes studied, the accuracy was not significant. Conclusion: Baseline frailty measured by the CFS is a good predictor of 90 and 180-day mortality and needing for home care in older adults admitted to ED. Its application in this setting might help clinical decision-making.

\section{INTRODUÇÃO}

Diversos fatores de risco têm sido identificados como preditores de mortalidade ${ }^{1}$ e declínio funcional em idosos admitidos em Serviços Hospitalares de Emergência (SHE), destacando-se a idade avançada e as doenças crônicas ${ }^{2}$. Entretanto, tais fatores, isoladamente, não são preditores suficientes de desfechos desfavoráveis em idosos em virtude da heterogeneidade dessa população ${ }^{3,4}$.

A Fragilidade constitui uma síndrome clínica complexa e multidimensional caracterizada por redução das reservas e da resistência ao estresse ${ }^{5}$, influenciada por fatores genéticos, ambientais, dietéticos e pela presença de doenças crônicas. Idosos frágeis, marcadamente em seu último ano de vida, são usuários frequentes dos serviços de atendimento préhospitalar ${ }^{6}, \mathrm{SHE}$, unidades de internação hospitalar e de terapia intensiva ${ }^{7}$. Nesses pacientes, a incidência de desfechos desfavoráveis é maior nos primeiros três meses após a alta hospitalar ${ }^{8}$. O grau de fragilidade basal de idosos admitidos em SHE é um preditor individual de mortalidade e de outros desfechos desfavoráveis?. A estratificação da fragilidade possibilita reconhecer pacientes para os quais tratamentos mais invasivos, possivelmente desproporcionais ao estado de saúde basal, podem ser evitados ${ }^{10}$.
Não há consenso quanto à melhor definição operacional de fragilidade ou escala mais apropriada para identificar idosos frágeis atendidos em SHE $\mathrm{SH}^{10-12}$. Neste cenário, as escalas devem ser de fácil e rápida aplicação e apresentar boa capacidade preditiva ${ }^{12,13}$. Algumas escalas de fragilidade utilizam medidas inviáveis para aplicação em SHE sobrelotados, onde os pacientes estão restritos ao leito e os profissionais pressionados pela necessidade de agilidade durante o atendimento ${ }^{10}$. Ademais, idosos robustos, quando gravemente enfermos e em situações de descompensação aguda de doenças crônicas, podem aparentar mais frágeis do que seu estado de saúde basal prévio à admissão ${ }^{12}$.

O declínio funcional é o principal marcador da fragilidade $^{14}$ e pode ser avaliado por instrumentos que estratificam o grau de fragilidade conforme o desempenho nas atividades de vida diária (AVDs). A Clinical Frailty Scale (CFS) ${ }^{14}$ considera a capacidade funcional para identificar e estratificar fragilidade. Foi validada em alguns países para predição de mortalidade em 30 e 90 dias da admissão no $\mathrm{SHE}^{15}$ e demonstrou boa capacidade preditiva de desfechos desfavoráveis em pacientes em situação de urgências clínicas e cirúrgicas admitidos no SHE e em terapia intensiva ${ }^{9,10,15-19}$. 
O National Institute for Health and Care Excellence (NICE) recomenda a aplicação da CFS a todos os idosos admitidos em caráter de urgência. Maior grau de fragilidade medido pela CFS em idosos admitidos no SHE associou-se ao aumento da taxa de mortalidade intra-hospitalar, variando de $2 \% \mathrm{em}$ idosos robustos até $24 \%$ em idosos com alto grau de fragilidade ${ }^{20}$. Estudos envolvendo idosos frágeis com covid-19 encontraram resultados similares. Contudo, as taxas de mortalidade encontradas foram mais elevadas $^{21,22}$. No Brasil, a CFS vem sendo estudada para estratificação de risco em pacientes com covid-1923. Entretanto, não foi avaliada para predição de desfechos desfavoráveis em idosos admitidos em SHE por motivos clínicos ou cirúrgicos em geral.

O objetivo primário deste estudo foi avaliar a capacidade da fragilidade basal, medida pela CFS, em predizer mortalidade em 90 dias de idosos admitidos em um SHE público brasileiro. Secundariamente, avaliamos a capacidade da CFS de predizer, durante o período de acompanhamento, outros desfechos desfavoráveis como a mortalidade em 180 dias da admissão, readmissão no SHE, reinternação, declínio funcional e necessidade de atenção domiciliar (AD) após a alta hospitalar. Adicionalmente, analisamos a sobrevida em 90 dias conforme as categorias da CFS.

\section{MÉTODOS}

Desenvolvemos um estudo de coorte prospectivo envolvendo pacientes idosos admitidos no SHE do Hospital das Clínicas da Universidade Federal de Minas Gerais (HC-UFMG) durante dois períodos de inclusão: de dezembro de 2019 a maio de 2020 e de novembro de 2020 a janeiro de 2021. A interrupção da coleta foi necessária como resposta à reestruturação do hospital para atender às demandas ocasionadas pela pandemia da covid-19.

Todos os pacientes com 60 anos ou mais admitidos no SHE por urgências clínicas ou cirúrgicas, e que permaneceram durante pelo menos uma noite nessa unidade, foram elegíveis para participar do estudo. Idosos que preencheram os critérios de inclusão foram convidados a participar. Todos os participantes foram selecionados por conveniência pela pesquisadora, previamente treinada na aplicação, interpretação e compreensão da CFS. Após receberem explicações sobre a pesquisa, todos os participantes ou seus responsáveis (no caso de pacientes com incapacidade cognitiva) que aceitaram participar, assinaram o Termo de Consentimento Livre e Esclarecido (TCLE) e foram submetidos ao mesmo protocolo de estudo.

Foram excluídos pacientes que receberam alta do SHE ou foram transferidos para outras unidades (centro cirúrgico, unidades de internação hospitalar ou terapia intensiva) antes do convite para o estudo, pacientes incapazes de se comunicar (pacientes em processo ativo de morte ou pacientes com demência avançada desacompanhados ou sem um cuidador capaz de consentir e fornecer informações confiáveis), bem como pacientes que se recusaram a assinar o TCLE. Em conformidade com os protocolos sanitários adotados pelo HC-UFMG na pandemia, pacientes internados na unidade de isolamento respiratório não foram incluídos, a fim de reduzir a transmissão do novo coronavírus.

Dados sociodemográficos (idade, sexo, escolaridade, raça/cor) e clínico-funcionais (comorbidades, uso de medicamentos, funcionalidade, mobilidade e histórico de internações) foram coletados a partir de relatos do paciente. No caso de incapacidade cognitiva do participante, definida pela avaliação clínica e/ou por dados do prontuário, as informações foram obtidas dos cuidadores e confirmadas no prontuário eletrônico. O histórico de internação em outros hospitais foi questionado aos participantes ou responsáveis.

Fragilidade basal foi definida como o grau de fragilidade de duas semanas antes da admissão ou anterior ao adoecimento agudo que motivou a internação ${ }^{12}$. CFS $\geq 5$ foi considerada como ponto de corte para definição de fragilidade ${ }^{14}$. Utilizamos a versão textual da CFS traduzida para o português brasileiro ${ }^{24}$ para estratificar o estado de fragilidade basal. Os idosos foram classificados variando de muito ativo (nível 1) a doente terminal (nível 9).

As variáveis clínicas coletadas foram definidas a partir de itens que compõem o Criteria for Screening and Triaging to Appropriate aLternative care (CriSTAL) ${ }^{25}$, um sistema de pontuação desenvolvido com o 
objetivo de predizer mortalidade de curto prazo em idosos admitidos no SHE e que foi validado em alguns países. No Brasil, sua utilização vem sendo estudada no HC-UFMG. Conforme critérios adotados no CriSTAL, consideramos como neoplasia avançada doença metastática e/ou aquela refratária ao tratamento; doença renal crônica (DRC), a DRC em estágio 4 ou 5 (taxa de filtração glomerular < $30 \mathrm{~mL} / \mathrm{min} / 1.73 \mathrm{~m} 2$ pelo CKD-EPI); insuficiência cardíaca crônica classe funcional III ou IV da New York Heart Association (NYHA); doença pulmonar obstrutiva crônica (DPOC) Global Initiative For Chronic Obstructive Disease (GOLD) 3 ou 4; acidente vascular encefálico (AVE) agudo ou ataque isquêmico transitório (AIT), história de infarto agudo do miocárdio; hepatopatia moderada a grave: hepatite viral crônica, hepatite crônica de outra causa, hepatopatia alcoólica com ou sem cirrose, insuficiência hepática crônica, fibrose hepática, qualquer cirrose, com ou sem hipertensão portal e/ ou varizes, transplantado hepático, esquistossomose hepatoesplênica). Multimorbidade foi definida como a presença de duas ou mais doenças crônicas ${ }^{26}$, que foram classificadas conforme Classificação Internacional de Doenças (CID-10).

O acompanhamento pós-alta foi realizado por contato telefônico com os participantes ou seus responsáveis entre 3 e 4 meses após a admissão no SHE. Nosso objetivo foi a verificação dos desfechos mortalidade em 90 dias e os desfechos secundários. Um segundo contato telefônico entre 6 e 7 meses foi realizado para verificar o desfecho secundário mortalidade até 180 dias da admissão. Ocorreram atrasos na localização de alguns pacientes, que demandaram várias tentativas de contato telefônico. Entretanto, para os desfechos mortalidade em 90 e 180 dias, somente os óbitos ocorridos dentro do período de estudo foram computados.

Utilizamos um questionário de acompanhamento padronizado que incluiu a reclassificação do nível de fragilidade atual e a utilização de serviços de saúde após a alta hospitalar. Para pacientes falecidos, foram coletadas informações sobre data, local e causa do óbito registrados na Declaração de Óbito.

O desfecho primário foi mortalidade em 90 dias da admissão no SHE. O desfecho secundário foi a ocorrência de uma medida composta por pelo menos um dos seguintes: mortalidade em 180 dias da admissão, readmissão em SHE, reinternação, necessidade de AD após a alta hospitalar e declínio funcional.

O tamanho amostral de 201 pacientes foi calculado para garantir um poder estatístico de $80 \%$ a fim de verificar a hipótese da associação entre a variável independente binária fragilidade basal (CFS $\geq 5$ ) e o desfecho mortalidade em 90 dias, por meio de regressão logística binária. Considerou-se a prevalência de fragilidade basal de pacientes hospitalizados de $56,7 \% \%^{19}$ e um nível de significância de 0,05 . Estimou-se uma taxa de óbito em 90 dias de $9,5 \%{ }^{8}$, variando até $24,5 \%$, considerando uma precisão de $15 \%$, com base na variabilidade dessa estimativa encontrada na literatura $a^{1,8,27,28}$.

Para análise estatística descritiva, após a verificação de normalidade com o teste ShapiroWilk, as variáveis contínuas foram expressas como medidas de tendência central e de dispersão. As variáveis categóricas foram expressas como distribuição de frequência e os dados foram tabulados de acordo com o status de fragilidade. Os testes qui-quadrado e exato de Fisher foram aplicados para examinar as associações entre as variáveis explicativas e a mortalidade em 90 dias. Para verificar o efeito da fragilidade sobre a mortalidade em 90 dias, foi realizado um modelo multivariado de regressão logística binária. $\mathrm{O}$ modelo foi ajustado para variáveis potencialmente confundidoras, incluídas conforme relevância clínica, evidência na literatura e valor $p<0,20$ na análise univariada. No modelo final, todas as variáveis que apresentaram valor $p<0,05$ foram mantidas. Idade e sexo foram incluídos a despeito da significância estatística. A retenção dessas variáveis no modelo justificou-se pela conhecida associação de sexo feminino com maior fragilidade e de sexo masculino com piores desfechos ${ }^{10}$. A classificação da fragilidade para o modelo foi dicotômica. Medidas de associação foram expressas como odds ratio (OR) e intervalo de confiança (IC) de 95\%.

As propriedades clinicométricas da CFS foram avaliadas por meio da sensibilidade, especificidade e da área sob a curva Receiver Operating Characteristics 
(AUROC). Uma AUROC $\geq 0,80$ foi considerada como tendo boa acurácia preditiva.

As curvas de sobrevida de Kaplan-Meier foram calculadas usando o teste de Logrank, Breslow e Tarone-Wire para analisar se o risco de morrer em 90 dias aumentou com o maior grau de fragilidade basal. Para essa análise, os pacientes foram estratificados em cinco grupos conforme categorias da CFS: 1-3, robusto; 4, vulnerável ou pré-frágil; 5 , fragilidade leve; 6 a 8, fragilidade moderada, grave e muito grave e 9, doente terminal. Essa distribuição foi adotada considerando o grau de dependência funcional e AVDs utilizado pela escala.

Os dados foram computados na plataforma online Research Electronic Data Capture (REDCap) ${ }^{29}$. Os resultados foram apresentados seguindo a metodologia STROBE ${ }^{30}$.
O estudo foi aprovado pelo Comitê de Ética em Pesquisa da UFMG, sob o protocolo CAAE: 23649519.0.0000.5149 e está em conformidade com as Resoluções no 466/2012 e 510/2016 do Conselho Nacional de Saúde.

\section{RESULTADOS}

Durante o período de recrutamento, 577 indivíduos com 60 anos ou mais foram admitidos e permaneceram durante pelo menos uma noite no SHE, preenchendo os critérios de elegibilidade. Um total de 371 pacientes foram excluídos. As razões para a exclusão foram documentadas (Figura 1). Ao final do acompanhamento de 6 meses, todos os participantes, exceto dois, tiveram os desfechos secundários verificados.

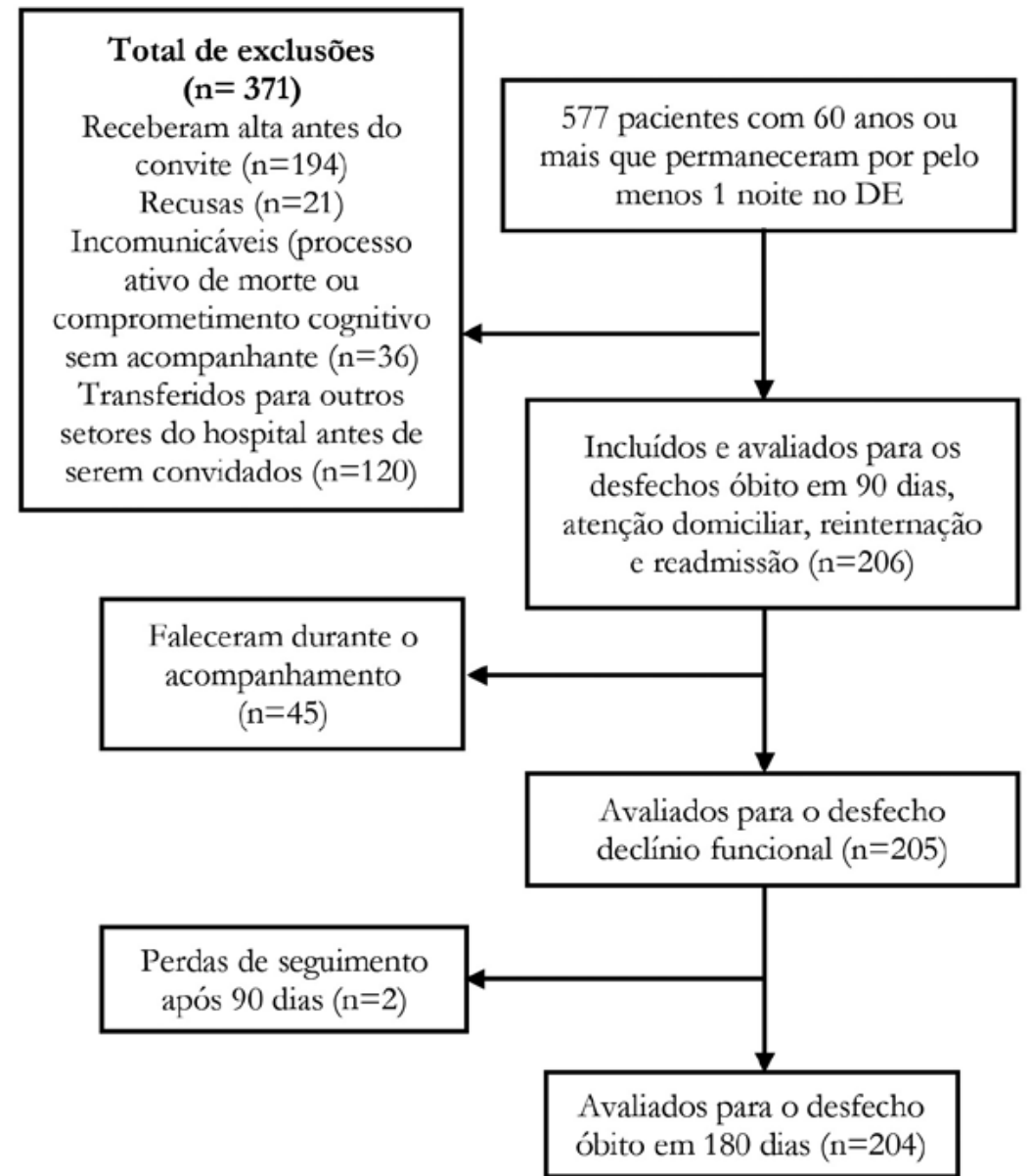

Figura 1. Fluxograma de seleção e acompanhamento dos participantes do estudo. Belo Horizonte, MG, 2019-2021. 
A mediana de idade foi 71 anos, intervalo interquartil (IIQ) de 66 a 78 anos, 87 pacientes $(42,2 \%)$ eram do sexo feminino. A prevalência de fragilidade na amostra foi de $61,7 \%$ (IC 95\% 54,6 a $68,3 \%$ ) e a mediana de pontuação da CFS foi 5 (IIQ 4-6). A maioria dos pacientes (96,7\%) foi admitida por motivo clínico. A associação entre a fragilidade e as e características dos pacientes e desfechos é apresentada na tabela 1. Os resultados sugerem que a fragilidade se associou a: sexo feminino, não morar sozinho, neoplasia avançada, AVE agudo ou AIT, presença de demência ou delirium à admissão e ter sido internado no último ano, inclusive em UTI.

Durante o período total de acompanhamento (mediana de 189 dias, mínimo de 2 e máximo de 226 dias), 61 (29,6\%) pacientes faleceram. Destes, 45 óbitos $(21,8 \%)$ ocorreram dentro de 90 dias da admissão no SHE. Não houve registro de óbito entre os participantes classificados como CFS 1 e 2. Observou-se um efeito dose-resposta entre fragilidade e mortalidade, onde a maior pontuação na CFS associou-se à maior mortalidade (CFS 3, 6\%; CFS 4, 7\%; CFS 5, 13\%; CFS 6, 20\%; CFS 7, 44\%; CFS $8,71 \%$ e CFS 9, 70\%) (Figura 2).

Houve uma forte associação positiva entre fragilidade basal e mortalidade em 90 dias da admissão no SHE (OR: 6,81; IC 95\% 2,55-18,13; $p<0,001)$. Após o ajuste para variáveis potencialmente confundidoras (idade, sexo, raça/cor, neoplasia avançada, hepatopatia, AVE agudo ou AIT, demência, delirium, multimorbidade, internação nos últimos 12 meses, UTI nos últimos 12 meses), a fragilidade medida pela CFS permaneceu no modelo como um forte preditor independente de mortalidade em 90 dias da admissão (OR: 3,84; IC 95\%: 1,36 a 10,90, $p=0,011)$. Permaneceram também no modelo final: neoplasia avançada, idade e UTI nos últimos 12 meses. Sexo masculino foi mantida no modelo devido a plausibilidade clínica, a despeito da significância estatística (Tabela 2). A performance da CFS para predizer todos os desfechos deste estudo foi apresentada como AUROC. A AUROC para mortalidade em 90 dias da admissão no SHE foi de 0,81 (IC 95\% 0,74-0,89; $p<0,001$ ), com sensibilidade de $88,9 \%$ e especificidade de $46 \%$ para o ponto de corte CFS $\geq 5$. Após ajuste, a CFS manteve boa capacidade preditiva (AUROC: 0,79; IC 95\% 0,71-0,86; $p<0,001$ ) (Tabela 2).

A análise da curva ROC para a CFS em relação à mortalidade em 180 dias mostrou uma AUROC de 0,80 , com IC $95 \%$ de 0,73 a $0,87(p<0,001)$. Para necessidade de AD, AUROC de 0,77 (IC 95\% de 0,69-0,86; $p<0,001)$ e reinternação, AUROC de 0,65 (IC $95 \%$ de $0,57-0,74 ; p=0,001$ ). Não encontramos associação entre a pontuação da CFS e readmissão em SHE (AUROC: 0,56, IC 95\% 0,46-0,66; $p=0,267$ ) ou declínio funcional (AUROC: 0,46, IC 95\% 0,37$0,54 ; p=0,325)$.

A Figura 3 apresenta as curvas de Kaplan-Meier pela CFS. Pelo valor $p$ referente ao teste de Logrank $(<0,001)$ e também aos testes de Breslow e TaroneWire, há evidências de que as curvas de sobrevivência se diferem com relação aos grupos da CFS. Pacientes classificados como CFS 9 apresentam uma tendência a falecer mais rapidamente que os demais, com aproximadamente $50 \%$ de probabilidade de falecer por volta do $37^{\circ}$ dia. As demais categorias seguem a ordem dos próprios grupos, coincidindo apenas o grupo robusto (1 a 3) com o grupo vulnerável (4). 
Tabela 1. Características dos pacientes e desfechos conforme status de fragilidade $(\mathrm{n}=206)$. Belo Horizonte, MG, 2019-2021.

\begin{tabular}{|c|c|c|c|c|}
\hline Variáveis & $\begin{array}{c}\text { CFS <5 } \\
(\mathrm{n}=79)\end{array}$ & $\begin{array}{l}\text { CFS } \geq 5 \\
(n=127)\end{array}$ & $\begin{array}{l}\text { Total } \\
(\mathrm{n}=206)\end{array}$ & Valor $p$ \\
\hline \multicolumn{5}{|l|}{ Características sociodemográficas } \\
\hline Idade, mediana (AIQ) & $70(64-76)$ & $71(66-79)$ & $71(66-78)$ & 0,106 \\
\hline \multicolumn{5}{|l|}{ Sexo, n $(\%)$} \\
\hline Feminino & $24(27,6)$ & $63(72,4)$ & $87(42,2)$ & 0,007 \\
\hline Masculino & $55(46,2)$ & $64(53,8)$ & $119(57,8)$ & \\
\hline \multicolumn{5}{|l|}{ Raça/ cor, n (\%) } \\
\hline Branca & $24(33,3)$ & $48(66,7)$ & $72(35,0)$ & 0,456 \\
\hline Parda & $41(42,3)$ & $56(57,7)$ & $97(47,1)$ & \\
\hline Negra & $11(34,4)$ & $21(65,6)$ & $32(15,5)$ & \\
\hline Indígena & $3(60,0)$ & $2(40,0)$ & $5(2,4)$ & \\
\hline \multicolumn{5}{|l|}{ Escolaridade, n (\%) } \\
\hline Analfabeto & $8(26,7)$ & $22(73,3)$ & $30(14,6)$ & 0,199 \\
\hline$<8$ anos & $49(37,4)$ & $82(62,6)$ & $131(63,6)$ & \\
\hline Entre 8 e 12 anos & $17(45,9)$ & $20(54,1)$ & $37(17,9)$ & \\
\hline$>12$ anos & $5(62,5)$ & $3(37,5)$ & $8(3,9)$ & \\
\hline Morar sozinho, n (\%) & $18(81,8)$ & $4(18,2)$ & $22(10,7)$ & $<0,001$ \\
\hline \multicolumn{5}{|l|}{ Características clínicas } \\
\hline Multimorbidade, $\mathrm{n}(\%)$ & $68(35,6)$ & $123(64,4)$ & $191(92,7)$ & 0,004 \\
\hline Neoplasia avançada (III/ IV), n (\%) & $4(9,5)$ & $38(90,5)$ & $42(20,4)$ & $<0,001$ \\
\hline DRC estágio 4 ou 5, n (\%) & $6(30,0)$ & $14(70,0)$ & $20(9,7)$ & 0,419 \\
\hline ICC (NYHA III / IV), n (\%) & $17(44,7)$ & $21(55,3)$ & $38(18,4)$ & 0,370 \\
\hline DPOC GOLD 3 ou 4, n (\%) & $2(22,2)$ & $7(77,8)$ & $9(4,4)$ & 0,487 \\
\hline AVE ou AIT atual, n (\%) & $14(66,6)$ & $8(33,4)$ & $22(10,7)$ & 0,010 \\
\hline História de IAM, n (\%) & $21(46,7)$ & $24(53,3)$ & $45(21,8)$ & 0,194 \\
\hline Hepatopatia, n (\%) & $3(18,8)$ & $13(81,2)$ & $16(7,7)$ & 0,093 \\
\hline Demência, n (\%) & $0(0,0)$ & $24(100,0)$ & $24(11,6)$ & $<0,001$ \\
\hline Delirium, n (\%) & $0(0,0)$ & $24(100,0)$ & $24(11,6)$ & 0,004 \\
\hline Internação nos últimos 12 meses, n (\%) & $44(30,6)$ & $100(69,4)$ & $144(69,9)$ & $<0,001$ \\
\hline UTI nos últimos 12 meses, n (\%) & $15(25,0)$ & $45(75,0)$ & $60(29,1)$ & 0,012 \\
\hline \multicolumn{5}{|l|}{ Desfechos } \\
\hline Mortalidade em 90 dias ( $\mathrm{n}=206), \mathrm{n}(\%)$ & $5(11,1)$ & $40(88,9)$ & $45(21,8)$ & $<0,001$ \\
\hline Mortalidade em 180 dias $(n=204), n(\%)$ & $9(14,8)$ & $52(85,2)$ & $61(29,9)$ & $<0,001$ \\
\hline Atenção domiciliar (n=206), n (\%) & $1(4,5)$ & $21(95,5)$ & $22(10,7)$ & $<0,001$ \\
\hline Reinternação ( $\mathrm{n}=206), \mathrm{n}(\%)$ & $13(22,4)$ & $45(77,6)$ & $58(28,2)$ & 0,003 \\
\hline Readmissão em SHE (n=206), n (\%) & $7(21,9)$ & $25(78,1)$ & $32(15,5)$ & 0,037 \\
\hline Declínio funcional (n=205), n (\%) & $28(43,7)$ & $36(56,3)$ & $64(31,2)$ & 0,301 \\
\hline
\end{tabular}

CFS = Clinical Frailty Scale; DRC = doença renal crônica; ICC =insuficiência cardíaca crônica;

CF NYHA =classe funcional da New York Heart Association; DPOC =doença pulmonar obstrutiva crônica; GOLD = Global Initiative For Chronic Obstructive Disease; AVE =acidente vascular encefálico; AIT =ataque isquêmico transitório; UTI =Unidade de Terapia Intensiva; SHE =Serviço Hospitalar de Emergência. 


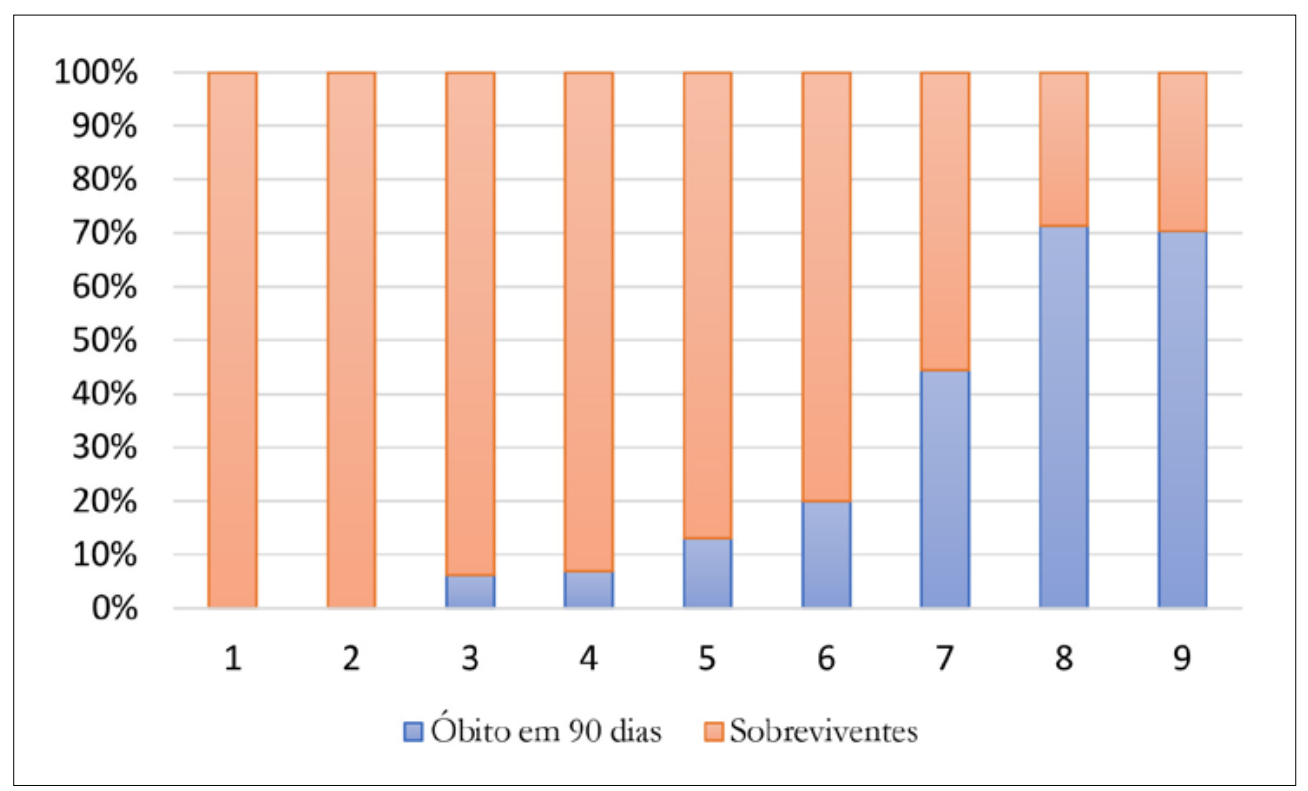

Figura 2. Mortalidade em 90 dias de acordo com a categoria da CFS (n=206). Belo Horizonte, MG, 2019-2021.

Tabela 2. Associação entre a fragilidade basal (CFS $\geq 5$ ) e óbito em 90 dias da admissão no Serviço Hospitalar de Emergência (SHE). Modelo final de regressão logística. Belo Horizonte, MG, 2019-2021.

\begin{tabular}{lll}
\hline Preditor & OR (IC 95\%) & Valor- $p$ \\
\hline Fragilidade ajustada & $3,84(1,36-10,90)$ & 0,011 \\
Neoplasia avançada & $4,11(1,79-9,47)$ & 0,001 \\
Idade, por ano & $1,06(1,01-1,10)$ & 0,019 \\
UTI nos últimos 12 meses & $2,51(1,15-5,48)$ & 0,021 \\
Sexo masculino & $1,36(0,64-2,91)$ & 0,425 \\
\hline
\end{tabular}

CFS = Clinical Frailty Scale; $\mathrm{AUROC}=$ area under Receiver Operating Characteristics curve; $\mathrm{UTI}=$ Unidade de Terapia Intensiva. 


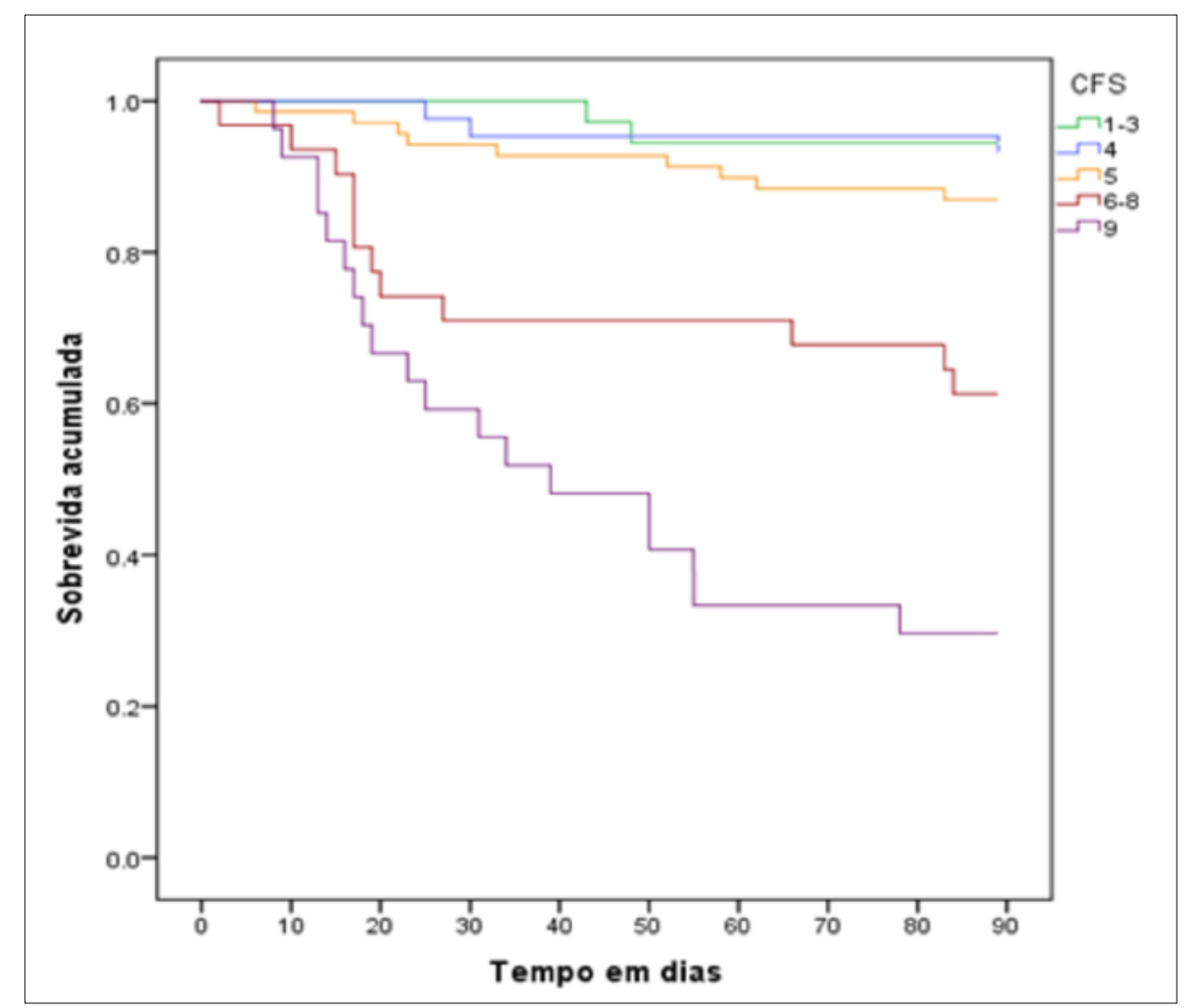

Figura 3. Curvas de sobrevida de Kaplan-Meier ao longo de 90 dias de acordo com cinco grupos da CFS. Belo Horizonte, MG, 2019-2021.

\section{DISCUSSÃO}

Este é o primeiro estudo brasileiro que utilizou a CFS para estratificar fragilidade em idosos atendidos no SHE por razões clínicas ou cirúrgicas, com o objetivo de avaliar sua capacidade de predizer desfechos desfavoráveis. Os resultados do presente estudo demostraram que a fragilidade basal, medida pela CFS, é um bom preditor de mortalidade em 90 dias da admissão no SHE. A capacidade preditiva da escala para o desfecho mortalidade em 90 e 180 dias em nossa amostra é comparável à encontrada por outros estudos que utilizaram a $\mathrm{CFS}^{10,27}$ e foi superior a outras escalas de fragilidade ${ }^{8,28}$. A CFS é uma escala válida e confiável ${ }^{15,31}$, de rápida e fácil aplicação que identifica e estratifica adequadamente a fragilidade. Observamos também um efeito dose-resposta entre a mortalidade em 90 dias e maior pontuação na CFS, semelhante ao demonstrado anteriormente ${ }^{15,32}$.
A prevalência de fragilidade em idosos hospitalizados observada em nossa amostra (61,7\%) está dentro da ampla faixa descrita na literatura (27 a $80 \%)^{9}$. Essa grande variabilidade se deve, em parte, ao instrumento de fragilidade utilizado. Quando comparada a estudos que utilizaram a CFS para estratificar idosos no SHE (entre 25 e 57\%) ) $^{15,16,18,19,27}$, a prevalência encontrada em nossa amostra foi um pouco superior. Encontramos também uma taxa de mortalidade superior às previamente descritas. Uma das hipóteses que podem justificar relaciona-se ao case mix do SHE do HC-UFMG, onde predominam atendimentos de pacientes com doenças crônicas graves e avançadas e que costumam ser mais frágeis do que pacientes atendidos em serviços hospitalares gerais, por trauma, ou casos de menor complexidade. Outra possibilidade foi o impacto da pandemia da covid-19, que contribuiu substancialmente para a maior mortalidade entre idosos. Alguns desses 
pacientes foram infectados durante o período de acompanhamento e faleceram por essa causa.

Podemos hipotetizar que a combinação da CFS com critérios clínicos de deterioração aguda, como escores de alerta precoce, venha a desempenhar melhor estratificação de risco em pacientes idosos admitidos no SHE do que a utilização dessas avaliações isoladamente, conforme demonstrado previamente $e^{23,33}$.

$\mathrm{Na}$ avaliação da capacidade preditiva da CFS para os demais desfechos desfavoráveis, a fragilidade basal demonstrou um desempenho moderado para predizer a necessidade de atenção domiciliar após a alta. Metade dos pacientes que necessitaram de atenção domiciliar foram classificados como CFS 8 e 9. Ressaltamos que, em nosso município, não dispomos de bospices. Contudo, os cuidados paliativos podem ser oferecidos para estes pacientes por serviços públicos de atenção domiciliar. Não encontramos estudos em língua inglesa ou portuguesa que avaliaram a capacidade da fragilidade medida pela CFS de predizer a necessidade de atenção domiciliar no curto prazo.

Conduzimos este estudo em situações reais, em um SHE de hospital público universitário de nível terciário no Brasil. $\mathrm{Na}$ realidade brasileira, assim como ocorre em outros países de baixa e média renda, essas unidades operam com lotação acima de sua capacidade, possuem longo tempo de espera para internação e grande demanda por leitos de internação hospitalar ${ }^{34}$. Os resultados observados no presente estudo sinalizam para a necessidade de agregar a identificação da fragilidade na rotina da avaliação de idosos no SHE. Além disso, no âmbito da gestão dos recursos públicos de saúde, o reconhecimento do estado de fragilidade da população auxilia no planejamento de políticas públicas, na organização da demanda por serviços especializados, na otimização dos programas de saúde e na melhor alocação dos recursos ${ }^{35}$.

Prognosticar é uma tarefa complexa, mas necessária e urgente no contexto atual. A avaliação do grau de fragilidade basal, por meio de escalas simples e rápidas, como a CFS, pode reduzir a incerteza prognóstica em cenários de mudanças rápidas no estado de saúde - como em unidades de emergências. Idosos muito frágeis são readmitidos em SHE, notadamente em seu último ano de vida, e são submetidos a tratamentos não benéficos ou inadequados ao seu estado de fragilidade basal. Esses tratamentos podem ter impacto negativo sobre a qualidade de vida do paciente e aumento dos custos emocionais e financeiros. Além disso, podem levar ao prolongamento desnecessário do processo de morrer, gerando falsas expectativas em pacientes e familiares, além de luto complicado.

A CFS poderia ser utilizada em SHE como um instrumento de triagem para identificar pacientes que se beneficiariam de uma avaliação geriátrica e gerontológica ampla e aqueles que não se beneficiariam de tratamentos de sustentação da vida. Isso possibilitaria o início de discussões oportunas com pacientes e familiares sobre os objetivos do cuidado e o planejamento pós-alta.

Nosso estudo tem algumas limitações. Devido a recursos humanos limitados para a coleta de dados, a inclusão foi realizada somente entre 8 e $17 \mathrm{~h}$ em dias úteis, o que limitou o tamanho amostral e levou a um potencial viés de seleção. Os pacientes que receberam alta do SHE no mesmo dia da admissão não foram incluídos. Presume-se que eles possam ser menos frágeis, considerando a curta permanência no SHE. Entretanto, não é possível generalizar os resultados para esses pacientes. A gravidade dos pacientes internados no HC-UFMG, um centro acadêmico de atenção terciária e quaternária, especialmente para câncer e doenças cardíacas, limita a generalização de nossos resultados para serviços de baixa complexidade. Pacientes incapazes de se comunicar ou aqueles em processo ativo de morte não foram incluídos no estudo, portanto, não é possível generalizar os resultados para esses grupos de pacientes.

\section{CONCLUSÃO}

Este estudo prospectivo mostrou que a fragilidade basal (relativa a duas semanas antes da admissão no Serviço Hospitalar de Emergência), mensurada pela Clinical Frailty Scale, foi capaz de identificar idosos com maior risco de morrer em curto prazo e de 
necessitar de serviços de atenção domiciliar. A Clinical Frailty Scale é uma escala válida, confiável e prática para predizer desfechos desfavoráveis em idosos atendidos no Serviço Hospitalar de Emergência. Sua utilização para estratificar o grau de fragilidade em idosos pode auxiliar na tomada de decisões e reduzir a incerteza prognóstica. Outros estudos são necessários para melhor esclarecer o papel da fragilidade e o impacto das condições agudas na mortalidade e outros desfechos desfavoráveis em curto e médio prazo em pacientes idosos atendidos em SHE no Brasil.

Editado por: Maria Helena Rodrigues Galvão

\section{REFERÊNCIAS}

1. Hofman SE, Lucke JA, Heim N, de Gelder J, Fogteloo AJ, Heringhaus C, et al. Prediction of 90-day mortality in older patients after discharge from an emergency department: a retrospective follow-up study. BMC Emerg Med [Internet]. 2016;16(1):1-9. Disponível em: https://doi. org/10.1186/s12873-016-0090-5 .

2. Kennelly SP, Drumm B, Coughlan T, Collins R, O’Neill D, Romero-Ortuno R. Characteristics and outcomes of older persons attending the emergency department: a retrospective cohort study. QJM [Internet]. 2014;107(12):977-87. Disponível em: https:// doi.org/10.1093/qjmed/hcu111 .

3. Chang AY, Skirbekk VF, Tyrovolas S, Kassebaum NJ, Dieleman JL. Measuring population ageing: an analysis of the Global Burden of Disease Study 2017. Lancet Public Health [Internet]. 2019;4(3):15967. Disponível em: http://doi.org/10.1016/S24682667(19)30019-2 .

4. Clegg A, Young J, Iliffe S, Rikkert MO, Rockwood K. Frailty in elderly people. Lancet [Internet]. 2013;381(9868):752-62. Disponível em: http://doi. org/10.1016/S0140-6736(12)62167-9 .

5. Rodríguez-Mañas L, Féart $\mathrm{C}$, Mann G, Viña J, Chatterji S, Chodzko-Zajko W, et al. Searching for an operational definition of frailty: A delphi method based consensus statement. the frailty operative definition-consensus conference project. J Gerontol Ser A Biol Sci Med Sci [Internet]. 2013;68(1):62-7. Disponível em: http://doi. org/10.1093/gerona/gls119 .

6. Lowthian JA, Jolley DJ, Curtis AJ, Currell A, Cameron PA, Stoelwinder JU, et al. The challenges of population ageing - accelerating demand emergency ambulance services by older. Med J Aust [Internet]. 2011:194(11):574-8. Disponível em: http:// doi.org/10.5694/j.1326-5377.2011.tb03107.x .

7. Ní Chróinín D, Goldsbury DE, Beveridge A, Davidson PM, Girgis A, Ingham N, et al. Healthservices utilisation amongst older persons during the last year of life: a population-based study. BMC Geriatr [Internet]. 2018;18(1):1-10. Disponível em: https://doi. org/10.1186/s12877-018-1006-x .

8. de Gelder J, Lucke JA, de Groot B, Fogteloo AJ, Anten S, Mesri K, et al. Predicting adverse health outcomes in older emergency department patients: the APOP study. Neth J Med. 2016;74(8):342-52.

9. Kahlon S, Pederson J, Majumdar SR, Belga S, Lau $\mathrm{D}$, Fradette M, et al. Association between frailty and 30-day outcomes after discharge from hospital. CMAJ [Internet]. 2015;187(11):799-804. Disponível em: http://doi.org/10.1503/cmaj.150100 .

10. Lewis ET, Dent E, Alkhouri H, Kellett J, Williamson $\mathrm{M}$, Asha S, et al. Which frailty scale for patients admitted via Emergency Department? A cohort study. Arch Gerontol Geriatr [Internet]. 2019;80:10414. Disponível em: http://doi.org/10.1016/j. archger.2018.11.002 .

11. Faller JW, Pereira DDN, Souza S, Nampo FK, Orlandi FS, Matumoto S. Instruments for the detection of frailty syndrome in older adults: a systematic review. PLoS ONE [Internet]. 2019;14(4):e0216166. Disponível em: http://doi. org/10.1371/journal.pone.0216166 .

12. Theou O, Campbell S, Malone ML, Rockwood K. Older Adults in the Emergency Department with Frailty. Clin Geriatr Med [Internet]. 2018;34(3):36986. Disponível em: http://doi.org/10.1016/j. cger.2018.04.003

13. O’Caoimh R, Costello M, Small C, Spooner L, Flannery A, O'Reilly L, et al. Comparison of frailty screening instruments in the emergency department. Int J Environ Res Public Health [Internet]. 2019;16(19):1-10. Disponível em: http://doi.org/10.3390/ijerph16193626 . 
14. Rockwood K, Song X, MacKnight C, Bergman $\mathrm{H}$, Hogan DB, McDowell I, et al. A global clinical measure of fitness and frailty in elderly people. CMAJ [Internet]. 2005;173(5):489-95. Disponível em: https:// doi.org/10.1503/cmaj.050051 .

15. Kaeppeli T, Rueegg M, Dreher-Hummel T, Brabrand M, Kabell-Nissen S, Carpenter CR, et al. Validation of the Clinical Frailty Scale for Prediction of Thirty-Day Mortality in the Emergency Department. Ann Emerg Med [Internet]. 2020;76(3):291-300. Disponível em: https://doi. org/10.1016/j.annemergmed.2020.03.028 .

16. Arteaga AS, Aguilar LT, González JT, Boza AS, Muñoz-Cruzado VD, Ciuró FP, et al. Impact of frailty in surgical emergencies: a comparison of four frailty scales. Eur J Trauma Emerg Surg [Internet]. 2021;47(5):16139. Disponível em: https://doi.org/10.1007/s00068020-01314-3 .

17. Guidet B, de Lange DW, Boumendil A, Leaver S, Watson X, Boulanger $\mathrm{C}$, et al. The contribution of frailty, cognition, activity of daily life and comorbidities on outcome in acutely admitted patients over 80 years in European ICUs: the VIP2 study. Intensive Care Med [Internet]. 2020;46(1):5769. Disponível em: https://doi.org/10.1007/s00134019-05853-1 .

18. Hewitt J, Carter B, McCarthy K, Pearce L, Law $\mathrm{J}$, Wilson FV, et al. Frailty predicts mortality in all emergency surgical admissions regardless of age. An observational study. Age Ageing [Internet]. 2019;48(3):388-94. Disponível em: https://doi. org/10.1093/ageing/afy217 .

19. Wallis SJ, Wall J, Biram RWS, Romero-Ortuno R. Association of the clinical frailty scale with hospital outcomes. QJM [Internet]. 2015;108(12):943-9. Disponível em: https://doi.org/10.1093/qjmed/ hev066.

20. National Health Service. Acute Frailty Network: Clinical Frailty Scale [Internet]. 2015 [acesso em 28 out. 2021]. Disponível em: https://www. acutefrailtynetwork.org.uk/Clinical-Frailty-Scale .

21. Chinnadurai R, Ogedengbe O, Agarwal P, MoneyCoomes S, Abdurrahman AZ, Mohammed S, et al. Older age and frailty are the chief predictors of mortality in COVID-19 patients admitted to an acute medical unit in a secondary care setting- a cohort study. BMC Geriatr [Internet]. 2020;20(1):19. Disponível em: https://doi.org/10.1186/s12877020-01803-5.
22. Hewitt J, Carter B, Vilches-Moraga A, Quinn TJ, Braude P, Verduri A, et al. The effect of frailty on survival in patients with COVID-19 (COPE): a multicentre, European, observational cohort study. Lancet Public Health [Internet]. 2020;5(8):44451. Disponível em: https://doi.org/10.1016/S24682667(20)30146-8 .

23. Aliberti MJR, Szlejf C, Avelino-Silva VI, Suemoto CK, Apolinario D, Dias MB, et al. COVID-19 is not over and age is not enough: Using frailty for prognostication in hospitalized patients. J Am Geriatr Soc [Internet]. 2021;69(5):111627. Disponível em: https://doi.org/10.1111/jgs.17146 .

24. Rodrigues MK, Nunes Rodrigues I, da Silva DJVG, Pinto JMS, Oliveira MF. Clinical Frailty Scale: Translation and Cultural Adaptation into the Brazilian Portuguese Language. J Frailty Aging [Internet]. 2021;10(1):38-43. Disponível em: https:// doi.org/10.14283/jfa.2020.7 .

25. Cardona-Morrell M, Hillman K. Development of a tool for defining and identifying the dying patient in hospital: Criteria for Screening and Triaging to Appropriate aLternative care (CriSTAL). BMJ Support Palliat Care [Internet]. 2015;5(1):7890. Disponível em: https://doi.org/10.1136/ bmjspcare-2014-000770 .

26. van den Akker M, Buntinx F, Metsemakers JFM, Roos S, Knottnerus JA. Multimorbidity in General Practice: Prevalence, Incidence, and Determinants of Co-Occurring Chronic and Recurrent Diseases. J Clin Epidemiol [Internet]. 1998;51(5):367-75. Disponível em: https://doi.org/10.1016/s0895-4356(97)00306-5 .

27. Cardona M, Lewis ET, Kristensen MR, SkjotArkil H, Ekmann AA, Nygaard HH, et al. Predictive validity of the CriSTAL tool for short-term mortality in older people presenting at Emergency Departments: a prospective study. Eur Geriatr Med [Internet]. 2018;9(6):891-901. Disponível em: https:// doi.org/10.1007/s41999-018-0123-6 .

28. Schuijt HJ, Oud FMM, Bruns EJR, van Duijvendijk P, Van der Zaag-Loonen HJ, Spies PE, van Munster BC. Does the Dutch Safety Management Program predict adverse outcomes for older patients in the emergency department? Neth J Med. 2020;78(5):244-50.

29. Harris PA, Taylor R, Minor BL, Elliott V, Fernandez $\mathrm{M}$, O'Neal L, et al. The REDCap consortium: Building an international community of software platform partners. J Biomed Inform [Internet]. 2019;95:1-9. Disponível em: https://doi. org/10.1016/j.jbi.2019.103208 . 
30. Malta M, Cardoso LO, Bastos FI, Magnanini MMF, Silva CMFP. Iniciativa STROBE: subsídios para a comunicação de estudos observacionais. Rev Saúde Pública [Internet]. 2010;44(3):559-65.

Disponível em: https://doi.org/10.1590/S003489102010000300021 .

31. Theou O, Pérez-Zepeda MU, van der Valk AM, Searle SD, Howlett SE, Rockwood K. A classification tree to assist with routine scoring of the Clinical Frailty Scale. Age Ageing [Internet]. 2021;50(4):140611. Disponível em: https://doi.org/10.1093/ageing/ afab006 .

32. Elliott A, Taub N, Banerjee J, Aijaz F, Jones W, Teece $\mathrm{L}$, et al. Does the Clinical Frailty Scale at Triage Predict Outcomes From Emergency Care for Older People? Ann Emerg Med [Internet]. 2021;77(6):620-

7. Disponível em: https://doi.org/10.1016/j. annemergmed.2020.09.006 .
33. Pulok MH, Theou O, van der Valk AM, Rockwood K. The role of illness acuity on the association between frailty and mortality in emergency department patients referred to internal medicine. Age Ageing [Internet]. 2020 ;49(6):10719. Disponível em: https://doi.org/10.1093/ageing/ afaa089.

34. Bittencourt RJ, Stevanato AM, Bragança CTNM, Gottems LBD, O'Dwyer G. Interventions in overcrowding of emergency departments: an overview of systematic reviews. Rev Saúde Pública [Internet]. 2020;54:1-9. Disponível em: http://doi.org/10.11606/ s1518-8787.2020054002342 .

35. Rockwood K, Theou O. Using the clinical frailty scale in allocating scarce health care resources. Can Geriatr J [Internet]. 2020;23(3)1-10. Disponível em: https://doi.org/10.5770/cgj.23.463 . 